\title{
Mechanism of Impaired Insulin-stimulated Muscle Glucose Metabolism in Subjects with Insulin-dependent Diabetes Mellitus
}

\author{
Gary W. Cline, Inger Magnusson, Douglas L. Rothman, Kitt Falk Petersen, Didier Laurent, and Gerald I. Shulman \\ Department of Internal Medicine, Yale University School of Medicine, New Haven, Connecticut 06520
}

\begin{abstract}
To determine the mechanism of impaired insulin-stimulated muscle glycogen metabolism in patients with poorly controlled insulin-dependent diabetes mellitus (IDDM), we used ${ }^{13} \mathrm{C}$-NMR spectroscopy to monitor the peak intensity of the $\mathrm{C} 1$ resonance of the glucosyl units in muscle glycogen during a 6-h hyperglycemic-hyperinsulinemic clamp using $\left[1-{ }^{13} \mathrm{C}\right]$ glucose-enriched infusate followed by nonenriched glucose. Under similar steady state $(t=3-6 \mathrm{~h})$ plasma glucose $(\sim 9.0 \mathrm{mM})$ and insulin concentrations $(\sim 400 \mathrm{pM})$, nonoxidative glucose metabolism was significantly less in the IDDM subjects compared with age-weight-matched control subjects $(37 \pm 6 \mathrm{vs}$. $73 \pm 11 \mu \mathrm{mol} / \mathrm{kg}$ of body wt per minute, $P<0.05$ ), which could be attributed to an $\sim 45 \%$ reduction in the net rate of muscle glycogen synthesis in the IDDM subjects compared with the control subjects $(108 \pm 16$ vs. $195 \pm 6 \mu \mathrm{mol} / \mathrm{liter}$ of muscle per minute, $P<0.001)$. Muscle glycogen turnover in the IDDM subjects was significantly less than that of the controls (16 \pm 4 vs. $33 \pm 5 \%, P<$ $0.05)$, indicating that a marked reduction in flux through glycogen synthase was responsible for the reduced rate of net glycogen synthesis in the IDDM subjects. ${ }^{31} \mathrm{P}-\mathrm{NMR}$ spectroscopy was used to determine the intramuscular concentration of glucose-6-phosphate (G-6-P) under the same hyperglycemic-hyperinsulinemic conditions. Basal G-6-P concentration was similar between the two groups $(\sim 0.10$ $\mathrm{mmol} / \mathrm{kg}$ of muscle) but the increment in G-6-P concentration in response to the glucose-insulin infusion was $\sim 50 \%$ less in the IDDM subjects compared with the control subjects $(0.07 \pm 0.02 \mathrm{vs} .0 .13 \pm 0.02 \mathrm{mmol} / \mathrm{kg}$ of muscle, $P<0.05)$. When nonoxidative glucose metabolic rates in the control subjects were matched to the IDDM subjects, the increment in the G-6-P concentration $(0.06 \pm 0.02 \mathrm{mmol} / \mathrm{kg}$ of muscle) was no different than that in the IDDM subjects. Together, these data indicate that defective glucose transport/phosphorylation is the major factor responsible for the lower rate of muscle glycogen synthesis in the poorly controlled insulin-dependent diabetic subjects. (J. Clin. Invest. 1997. 99:2219-2224.) Key words: muscle • diabetes • glycogen • glucose-6-phosphate - nuclear magnetic resonance spectroscopy
\end{abstract}

Address correspondence to Gerald I. Shulman, M.D., Ph.D., Yale University, School of Medicine, Department of Internal Medicine, Fitkin 1, P.O. Box 20820, New Haven, CT 06520-8020. Phone: 203785-5447; FAX: 203-785-6015; E-mail: Gerald_Shulman@QM.yale.edu

Received for publication 23 April 1996 and accepted in revised form 20 February 1997.

J. Clin. Invest.

(C) The American Society for Clinical Investigation, Inc.

0021-9738/97/05/2219/06 \$2.00

Volume 99, Number 9, May 1997, 2219-2224

\section{Introduction}

Peripheral insulin resistance can be correlated to glycemic control in patients with poorly controlled insulin-dependent diabetes mellitus (IDDM) ${ }^{1}$ however, the underlying mechanisms of this apparent glucose toxicity are unclear (1-3). One possible explanation for hyperglycemia per se to result in insulin resistance may be the desensitization of the glucose transport system by elevated levels of hexosamine, an alternate product of glucose metabolism. Glucosamine has been shown to interfere with the translocation of the GLUT4 glucose transporters in adipocytes in vitro (4), and in rat muscle in vivo (5). However, there is no direct evidence that this can account for the glucose toxicity observed in humans. Therefore, we have applied ${ }^{13} \mathrm{C}$ - and ${ }^{31} \mathrm{P}$-NMR spectroscopy combined with the glucose clamp technique to study muscle glucose metabolism in normal subjects and subjects with poorly controlled IDDM to determine whether glucose transport/phosphorylation can account for the impaired glucose uptake in IDDM subjects, as predicted by the hexosamine theory.

Rates of muscle glycogen synthesis were measured in subjects with poorly controlled IDDM and in age-weightmatched controls during a hyperglycemic-hyperinsulinemic clamp using ${ }^{13} \mathrm{C}$-NMR spectroscopy $(6,7)$. Relative rates of muscle glycogen turnover (i.e., simultaneous glycogen synthesis and degradation) were assessed by following the $\left[1-{ }^{13} \mathrm{C}\right]$ glucose infusion period with an infusion of natural abundance glucose (8). Intramuscular glucose-6-phosphate (G-6-P) concentration was measured using ${ }^{31} \mathrm{P}-\mathrm{NMR}$ under the same conditions to identify which enzymatic step can account for the reduced rate of muscle glycogen synthesis in the IDDM patients $(9,10)$. As an intermediate between glucose transport/phosphorylation and glycogen synthase, the correlation of the G-6-P concentration with glycogen synthesis rates serves as an index of the relative degree to which these two steps control the rate of glycogen synthesis.

\section{Methods}

Subjects. Six (four males, two females) lean (BMI, $24.2 \pm 0.8 \mathrm{~kg} / \mathrm{m}^{2}$ ) type I (IDDM) diabetic subjects (age $34 \pm 5 \mathrm{yr}$ ) were studied (protocols 1 and 2). All the diabetic subjects fulfilled the criteria for the diagnosis of diabetes mellitus as established by the National Diabetes Data Group. Subjects with IDDM were poorly controlled and had a mean glycosylated hemoglobin $\mathrm{A}_{1}$ of $13.6 \pm 1.4 \%$ (normal range, 4-8\%). No subject had any major disease other than diabetes mellitus or was taking medications other than insulin. Two groups (protocol 1: four males, two females; protocol 2: two males, eight females) of ageweight-height-matched (BMI, protocol 1: $21.6 \pm 0.8 \mathrm{~kg} / \mathrm{m}^{2}$, protocol 3: $23.5 \pm 0.6 \mathrm{~kg} / \mathrm{m}^{2}$ ) healthy subjects (ages, protocol $1: 29 \pm 2 \mathrm{yr}$, protocol 3: $30 \pm 3 \mathrm{yr}$ ) were studied. None of the normal subjects had a family history of diabetes.

1. Abbreviations used in this paper: G-6-P, glucose-6-phosphate; IDDM, insulin-dependent diabetes mellitus; RMS, root mean square. 
Informed consent was obtained from all subjects after the purpose, nature, and potential risks of the study were explained to them. The protocol was reviewed and approved by the Human Investigation Committee of the Yale University School of Medicine.

Experimental protocol. All studies were begun at 8 a.m. after an overnight fast of 10-12 h. $3 \mathrm{~d}$ before the study, all subjects were put on a weight-maintaining diet (prepared by the metabolic kitchen of the General Clinical Research Center) of $33 \mathrm{~g} / \mathrm{kg}$ body weight consisting of $50 \%$ carbohydrate, $20 \%$ protein, and $30 \%$ fat. To induce normoglycemia $(\sim 5.5 \mathrm{mmol} /$ liter $)$, all diabetic subjects received an overnight variable infusion of insulin $(0.6-1.5 \mathrm{pmol} / \mathrm{kg}$ of body weight per minute) through a Teflon catheter placed in an antecubital vein (7). The induction of euglycemia allowed the diabetic and normal subjects to be studied while they had the same plasma glucose concentrations and responded to an identical increment above baseline in the plasma glucose concentration. On the morning of the study a second Teflon catheter was inserted into the antecubital vein of the opposite arm to permit blood to be drawn. Intravenous catheters were inserted in each arm of the normal subjects on the morning of the study.

Measurements of glycogen synthesis and turnover rates by ${ }^{13} \mathrm{C}$ NMR spectroscopy (protocol 1) and G-6-P levels by ${ }^{31} \mathrm{P}-\mathrm{NMR}$ spectroscopy (protocols 2 and 3) were done on separate occasions. The IDDM subjects were each studied twice, and two groups of control subjects were studied (two of the control subjects participated in both protocols 1 and 3, data for seven of the control subjects in protocol 3 have been reported previously [10]).

Hyperglycemic-hyperinsulinemic clamp procedure. Hyperglycemichyperinsulinemia was induced with the insulin-glucose clamp technique as described previously (11). To inhibit endogenous insulin and glucagon secretion, an infusion of somatostatin $(0.1 \mu \mathrm{g} / \mathrm{kg}$ per minute) was initiated 5 min before the start of the glucose-insulin infusion in both the normal and diabetic subjects. At time 0 , insulin (Humulin-R; Eli Lilly and Co., Indianapolis, IN) was administered in a priming and continuous infusion of $240 \mathrm{pmol} / \mathrm{m}^{2}$ of body surface area per minute to raise the plasma insulin concentration acutely and maintain it at $\sim 400 \mathrm{pmol} /$ liter for the duration of the study. At the same time, one of three glucose infusion protocols was initiated.

Throughout the study, plasma glucose concentration was measured every $5 \mathrm{~min}$ and the infusion rate of the glucose solution was periodically adjusted to maintain the desired hyperglycemic plateau. Under these conditions of constant hyperglycemia and hyperinsulinemia, hepatic glucose production is completely suppressed and all infused glucose is taken up by tissue, except for a minor amount excreted in urine. The latter amount was measured and the rate of excretion was subtracted from the rate of glucose infusion. Therefore, the mean glucose infusion rate, minus urinary glucose excretion, serves as a measure of the total amount of glucose metabolized. Blood samples were taken for measurement of plasma glucose ${ }^{13} \mathrm{C}$ enrichment every $15 \mathrm{~min}$, plasma insulin concentration every $30 \mathrm{~min}$, and plasma lactate every $60 \mathrm{~min}$.

Protocol 1; Muscle glycogen synthesis and turnover in IDDM and control subjects. At time 0, a variable priming infusion of glucose $\left(1.11 \mathrm{M},\left[1-{ }^{13} \mathrm{C}\right]\right.$ glucose-enriched, $\left.10-20 \%{ }^{13} \mathrm{C}\right)$ was begun so that the plasma glucose concentration could be acutely raised and maintained at $\sim 9 \mathrm{mM}$ for the duration of the study. From 60 to $120 \mathrm{~min},{ }^{13} \mathrm{C}$ NMR was used to assess rates of liver glycogen synthesis in five of the IDDM and all six of the control subjects, as reported previously (7). At $240 \mathrm{~min}$, the infusate was switched to natural abundance $\left[{ }^{13} \mathrm{C}\right] \mathrm{glu}-$ cose $(1.11 \mathrm{M})$ to maintain hyperglycemia at $\sim 9 \mathrm{mM}$ for an additional $120 \mathrm{~min}$, while allowing plasma glucose ${ }^{13} \mathrm{C}$ atom percent excess to return to natural abundance. Muscle glycogen synthesis rates and turnover were assessed by ${ }^{13} \mathrm{C}-\mathrm{NMR}$ spectroscopy.

Protocol 2; Muscle G-6-P concentration in IDDM subjects. This protocol was designed to determine the incremental change in G-6-P associated with rates of net glycogen synthesis observed in protocol 1 in the IDDM subjects. At time 0 , a variable priming infusion of natural abundance glucose $(1.11 \mathrm{M})$ was begun so that the plasma glucose concentration could be acutely raised and maintained at $\sim 9 \mathrm{mM}$ for the duration of the study (120 min). Muscle phosphorus metabolites were assessed by ${ }^{31} \mathrm{P}-\mathrm{NMR}$ spectroscopy.

Protocol 3; Muscle G-6-P concentration in control subjects. This protocol was designed to correlate the incremental change in G-6-P associated with rates of net glycogen synthesis observed in protocol 1 in the control subjects, and under conditions in which the nonoxidative glucose disposal in the control subjects was matched to that of the IDDM subjects. At time 0 , a variable priming infusion of natural abundance glucose $(1.11 \mathrm{M})$ was begun so that the plasma glucose concentration could be acutely raised and maintained at $\sim 9 \mathrm{mM}$ for the duration of the study. At $120 \mathrm{~min}$, the glucose infusion rate was decreased to allow plasma glucose concentration to fall to euglycemic levels in order to match and maintain for $60 \mathrm{~min}$ the nonoxidative glucose disposal rates of the control subjects with that of the IDDM subjects during protocol 2 .

Measurements. Continuous indirect calorimetry was performed to determine whole body glucose oxidation as described (6-10). Nonoxidative glucose metabolism was calculated by subtracting the amount of glucose oxidized from the total amount of glucose infused.

Plasma glucose concentration was measured by the glucose oxidase method (Glucose Analyzer II; Beckman Instruments, Inc., Fullerton, $\mathrm{CA}$ ). ${ }^{13} \mathrm{C}$ atom percent enrichment of plasma glucose (pentaacetate) was determined by GC-MS, as described previously $(6,7)$.

NMR spectroscopic techniques. During the measurements, the subjects remained supine within an NMR spectrometer (Bruker Instruments, Inc., Billerica, MA; 1-m bore, 2.1T). The gastrocnemius muscle of the right leg was positioned within the homogeneous volume of the magnet on top of a concentric surface coil probe. The probe consisted of a 9-cm-diameter inner coil for ${ }^{31} \mathrm{P}$ or ${ }^{13} \mathrm{C}$ acquisition and a 13-cm outer coil for ${ }^{1} \mathrm{H}$ acquisition, decoupling, and shimming.

${ }^{13} \mathrm{C}$-NMR spectra were obtained with a pulse-acquire sequence in 10 -min blocks consisting of 4,800 scans using a $90^{\circ}$ pulse at coil center and a repetition time of $120 \mathrm{~ms}$. Decoupling at the ${ }^{1} \mathrm{H}$ frequency at a power of $15 \mathrm{~W}$ was applied at the glycogen $\mathrm{C} 1$ proton resonance frequency during the 25.6-ms acquisition period. Quantitation of the [1${ }^{13} \mathrm{C}$ ] glycogen concentration in glucosyl units was relative to a solution of oyster glycogen in a mold of the right calf of each subject (6). A 2-cm sphere containing $\left[{ }^{13} \mathrm{C}\right]$ formic acid was used as a pulse power and loading calibration. The ${ }^{13} \mathrm{C}$-NMR muscle glycogen measurement has been validated by comparison with biochemical assay of glycogen measured in biopsied human muscle tissue (12).

${ }^{31} \mathrm{P}-\mathrm{NMR}$ spectra were obtained using a $70^{\circ}$ pulse at coil center $(100 \mu \mathrm{s})$ with a repetition time of $2 \mathrm{~s}$ and a 256-ms acquisition time (9, 10). During acquisition, ${ }^{1} \mathrm{H}$ power $(1 \mathrm{~W})$ was applied at the position of the C6 protons of G-6-P to decouple the $J_{\mathrm{H}-\mathrm{P}}$ interaction. Spectra were acquired in 288 scans with 4,096 data points. Time domain data were zero filled and apodized using either a $2-$ or $10-\mathrm{Hz}$ exponential function and a mild $500-\mathrm{Hz}$ profile correction. Concentrations of metabolites were calculated from the data as described $(9,10)$. The area of the $\beta$ resonance of ATP was used as an internal concentration standard and was assumed to represent a concentration of $5.5 \mathrm{mmol} /$ $\mathrm{kg}$ of muscle $(9,10)$. Chemical shifts are referenced to $\mathrm{PCr}$ at 0.00 $\mathrm{ppm}$. The resonance for G-6-P is in a region of the ${ }^{31} \mathrm{P}-\mathrm{NMR}$ spectrum in close proximity to other phosphomonoesters resonances. Any potential contribution from other phosphomonoester resonances, which have chemical shifts upfield (lower ppm) to G-6-P, was minimized by integrating over the chemical shift range of the downfield half of the G-6-P resonance (7.43-7.13 ppm) and multiplying by two, as described previously $(9,10)$. The precision of the G-6-P concentration measurement was determined from the root mean square (RMS) amplitude variation in the NMR difference spectrum due to spectral noise (9). The RMS amplitude variation due to noise, which is independent of signal intensity, was calculated as one-fifth of the maximum peak to peak amplitude variation in a $1-\mathrm{kHz}$ bandwidth in the spectrum (100 times the G-6-P resonance bandwidth) not containing resonances. The average RMS noise would result in a $1 \mathrm{SD}$ variation 
in the measured G-6-P concentration of $0.015 \mathrm{mmol} / \mathrm{kg}$ of muscle in a 40-min difference spectrum. Variation in intensity due to spectrometer instability was a second potential source of error in the G-6-P measurement. Spectrometer stability was assessed from the residual intensity of the ATP resonances in each difference spectrum and the subtraction accuracy in all cases was better than $1 \%$. With this accuracy the measured G-6-P basal concentrations would result in a variation in intensity (IDDM: $<0.0011$; controls: $<0.0013 \mathrm{mmol} / \mathrm{kg}$ of muscle) $>10$-fold less than the variation due to RMS noise. The ${ }^{31} \mathrm{P}$ NMR G-6-P measurement has been validated in an animal model by comparison with chemical assay of G-6-P done on muscle frozen in situ (13).

Calculations. Increments in muscle glycogen concentration were calculated from the increase in $\left[{ }^{13} \mathrm{C}\right]$ glycogen concentration and the plasma $\left[1-{ }^{13} \mathrm{C}\right]$ glucose atom percent enrichment during the $\left[1-{ }^{13} \mathrm{C}\right]$ glucose infusion as described (6-8). Net rates of glycogen synthesis were calculated from the slope of the least-squares linear fit to their glycogen concentration curve from $t=150$ to $240 \mathrm{~min}$. Previously, we have equated the slope with the rate of glycogen synthesis, $V_{\text {in }}$, assuming that any concurrent glycogen breakdown would not result in loss of labeled glycogen, because the $\left[1-{ }^{13} \mathrm{C}\right]$ glycogen signal intensity was monitored during the initial period of glycogen repletion $(t=1-2 \mathrm{~h})$ when basal glycogen stores represented the majority of glycogen available for mobilization by glycogen phosphorylase. However, in this study, we did not begin monitoring changes in $\left[1-{ }^{13} \mathrm{C}\right]$ glycogen until $150 \mathrm{~min}$ after the start of the $\left[1-{ }^{13} \mathrm{C}\right]$ glucose infusion, when labeled glycogen could represent a significant fraction of glycogen present in the muscle. Therefore, under these conditions it is likely that both labeled and unlabeled glycogen is being lost during the measurement of the $\left[1-{ }^{13} \mathrm{C}\right]$ glycogen signal and that the calculated rate may therefore more closely approximate the net rate of glycogen synthesis, $V_{\text {net }}$ (where $V_{\text {net }}=V_{\text {in }}-V_{\text {out }}$ ), as opposed to the absolute rate of glycogen synthesis, $V_{\text {in }}$.

Previously, as given by Eq. 1, the minimal estimate of glycogen breakdown, $V_{\text {out }}$, during the chase period of unlabeled glucose was determined from the difference in the observed change of the [1$\left.{ }^{13} \mathrm{C}\right]$ glycogen concentration, $\Delta\left[{ }^{13} \mathrm{C}-\mathrm{Gly}\right]_{\mathrm{o}}$, and the predicted change of the $\left[1-{ }^{13} \mathrm{C}\right]$ glycogen concentration calculated from the time interval, $\Delta T$, the synthetic rate, $V_{\text {in }}$, and plasma glucose fractional enrichment, $f e_{\text {glc }}(8)$. As discussed, we have purposely calculated the minimal value of $V_{\text {out }}$ by assuming a maximum value for the $\left[1-{ }^{13} \mathrm{C}\right]$ glycogen fractional enrichment, $f e_{\text {gly }}$, equal to the maximum fractional enrichment of plasma glucose, $f e_{\text {glc }}(8)$ :

$V_{\text {out }}=\left(1 / f e_{\text {gly }}\right) \cdot\left(\Delta T \cdot V_{\text {in }} \cdot f e_{\text {glc }}-\Delta\left[{ }^{13} \mathrm{C}-\mathrm{Gly}\right]_{\mathrm{o}}\right)$.

However, with the consideration that our synthetic rate probably approximates $V_{\text {net }}$, as opposed to $V_{\text {in }}$, we derived Eq. 2 to calculate $V_{\text {out }}$ under these conditions:

$V_{\text {out }}=\left[1 /\left(f e_{\mathrm{gly}}-f e_{\mathrm{glc}}\right)\right] \cdot\left(\Delta T \cdot V_{\mathrm{net}} \cdot f e_{\mathrm{glc}}-\Delta\left[{ }^{13} \mathrm{C}-\mathrm{Gly}\right]_{\mathrm{o}}\right)$.

Glycogen turnover was calculated as:

Gly turnover $(\%)=100 \cdot\left(V_{\text {out }} / V_{\text {in }}\right)$.

Statistics. Measurements are given as the group mean \pm SEM. Statistical differences between groups were performed using the Student's $t$ test.

\section{Results}

Hyperglycemic-hyperinsulinemic clamp. Basal glucose and free insulin plasma levels were both slightly higher in the IDDM subjects $(6.6 \pm 0.5 \mathrm{mM}$ and $82 \pm 6 \mathrm{pM})$ compared with the control subjects $(5.2 \pm 0.2 \mathrm{mM}$ and $58 \pm 7 \mathrm{pM})(P<0.01)$. There were no differences in the basal concentrations of metabolites determined by ${ }^{31} \mathrm{P}-\mathrm{NMR}$ (G-6-P, $0.11 \pm 0.03$ and
$0.13 \pm 0.01 \mathrm{mmol} / \mathrm{kg}$ of muscle; $\mathrm{Pi}, 3.4 \pm 0.4$ and $3.0 \pm 0.2 \mathrm{mmol} /$ $\mathrm{kg}$ of muscle; $\mathrm{PCr}, 24.6 \pm 0.9$ and $23.2 \pm 0.7 \mathrm{mmol} / \mathrm{kg}$ of muscle; $\mathrm{pH} 7.05 \pm 0.01$ and $7.03 \pm 0.01 ; P=\mathrm{NS}$ for IDDM and controls, respectively). Unlike G-6-P and PCr which are confined to the intracellular space, the Pi signal observed in the ${ }^{31} \mathrm{P}$ spectra of the muscle tissue is from both intracellular and extracellular $\mathrm{Pi}$, thereby precluding any firm conclusions regarding changes in the intracellular phosphate concentration. Steady state for plasma glucose concentration was reached by $\sim 15 \mathrm{~min}$, and $\sim 90$ min for ${ }^{13} \mathrm{C}$ plasma glucose enrichment in both groups. Mean steady state plasma glucose concentration (IDDM, $9.0 \pm 0.1 \mathrm{mM}$; control, $8.9 \pm 0.2 \mathrm{mM}, P=\mathrm{NS}$ ) and free insulin concentration (IDDM, 402 $\pm 18 \mathrm{pM}$; control, $417 \pm 14 \mathrm{pM}, P=$ NS) were similar between the two groups. Baseline blood lactate concentration was similar in the two groups (IDDM, $0.59 \pm 0.04 \mu \mathrm{M}$; control, $0.65 \pm 0.07 \mu \mathrm{M}, P=\mathrm{NS}$ ) and increased significantly during the hyperglycemic-hyperinsulinemic clamp (IDDM, 0.91 $\pm 0.04 \mu \mathrm{M}$; control, $1.27 \pm 0.13 \mu \mathrm{M}$, both $P<0.05$ compared with basal, $P<0.05$ control vs. IDDM). During the clamp, both groups had an initial increase in rates of whole body glucose metabolism that plateaued by $180 \mathrm{~min}$ (Fig. 1). Whole body glucose metabolic rates were $\sim 40 \%$ less in the IDDM subjects compared with the controls at all times throughout the study $(P<0.05)$. During the time interval $(60$ $120 \mathrm{~min}$ ) for measurement of G-6-P concentrations under hyperglycemic-hyperinsulinemia, whole body glucose metabolic rates were not significantly different between study days (IDDM, $32 \pm 6$ vs. $34 \pm 5 \mu \mathrm{mol} / \mathrm{kg}$ of body weight per minute; controls, $57 \pm 4$ vs. $66 \pm 7 \mu \mathrm{mol} / \mathrm{kg}$ of body weight per minute, glycogen synthesis rates vs. G-6-P measurements, respectively). During euglycemic-hyperinsulinemia (protocol 3), mean whole body glucose metabolic rates fell to $41 \pm 3 \mu \mathrm{mol} /$ $\mathrm{kg}$ of body weight per minute in the control subjects. Rates of glycogen synthesis and turnover were measured during an interval of steady state whole body glucose metabolism; $57 \pm 6$ vs. $96 \pm 11 \mu \mathrm{mol} / \mathrm{kg}$ of body weight per minute from 180 to 240 $\mathrm{min}, 51 \pm 2 \mathrm{vs}$. $90 \pm 11 \mu \mathrm{mol} / \mathrm{kg}$ of body weight per minute from 300 to $360 \mathrm{~min}$ (IDDM vs. control subjects, respectively).

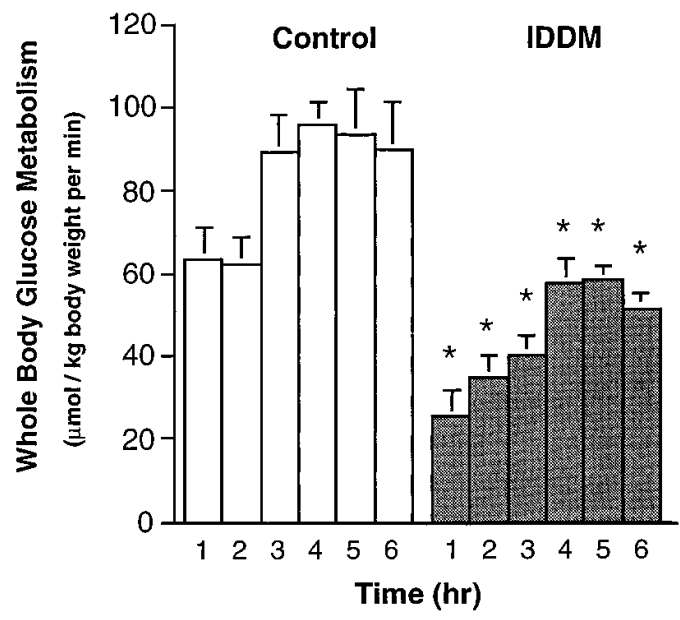

Figure 1. Mean rates of nonoxidative and oxidative whole body glucose metabolism in control subjects and subjects with poorly controlled IDDM during a 6-h hyperglycemic-hyperinsulinemic clamp. ${ }^{*} P<0.05$ (compared with the control subjects during the same time interval). 


\begin{tabular}{|c|c|c|c|c|c|c|c|c|}
\hline \multirow[b]{2}{*}{ Study group } & \multicolumn{3}{|c|}{ Glucose metabolism } & \multicolumn{3}{|c|}{ Muscle glycogen } & \multirow[b]{2}{*}{$\delta \mathrm{G}-6-\mathrm{P}$} & \multirow[b]{2}{*}{$\delta P_{i}$} \\
\hline & Total & Oxid & Nonoxid & $V_{\text {net }}$ & $V_{\text {out }}$ & $V_{\text {in }}$ & & \\
\hline & \multicolumn{3}{|c|}{ $\mu \mathrm{mol} / \mathrm{kg}$ body wt per minute } & \multicolumn{3}{|c|}{$\mu \mathrm{mol} /$ liter of muscle per minute } & \multicolumn{2}{|c|}{$\mathrm{mmol} / \mathrm{kg}$ of muscle } \\
\hline IDDM & $57 \pm 6$ & $20 \pm 2$ & $37 \pm 6$ & $108 \pm 16$ & $21 \pm 8$ & $129 \pm 24$ & $0.07 \pm 0.02$ & $1.10 \pm 0.70$ \\
\hline Controls & $96 \pm 11$ & $23 \pm 3$ & $73 \pm 11$ & $195 \pm 6$ & $96 \pm 19$ & $291 \pm 24$ & $0.13 \pm 0.02$ & $0.64 \pm 0.06$ \\
\hline$P$ value & $<0.05$ & NS & $<0.05$ & $<0.001$ & $<0.05$ & $<0.05$ & $<0.05$ & NS \\
\hline
\end{tabular}

Rates of glucose oxidation were similar in both groups and did not change appreciably during the clamp (controls, 23 \pm 3 $\mu \mathrm{mol} / \mathrm{kg}$ of body weight per minute; IDDM, $20 \pm 2 \mu \mathrm{mol} / \mathrm{kg}$ of body weight per minute, $P=\mathrm{NS}$ ).

Muscle glycogen synthesis. Glycogen synthesis rates were measured after the subjects had reached steady state rates of whole body glucose metabolism. By this time, there is considerable ${ }^{13} \mathrm{C}$ enrichment of the glycogen. In contrast to our earlier studies (6-8), turnover of glycogen would therefore result in loss of ${ }^{13} \mathrm{C}$ label simultaneous with net incorporation, and the observed change in the $\left[1-{ }^{13} \mathrm{C}\right]$ glycogen signal can best be equated with net glycogen synthesis, $V_{\text {net }}$. To determine $V_{\text {in }}$ under these conditions therefore, it is necessary to also determine $V_{\text {out }}$. The rate of $\left[1-{ }^{13} \mathrm{C}\right]$ glucose incorporation into $\mathrm{C} 1$ glycogen was linear in both groups (IDDM subjects, $r=0.944 \pm 0.031$; controls, $r=0.978 \pm 0.009)$ and the net rates of muscle glycogen synthesis were calculated to be $108 \pm 16 \mu \mathrm{mol} / \mathrm{liter}$ of muscle per minute in the IDDM group and $195 \pm 6 \mu \mathrm{mol} / \mathrm{liter}$ of muscle per minute in the control group $(P<0.001)$. Assuming that muscle mass is equivalent to $26 \%$ body weight (14), net muscle glycogen synthesis could account for $76 \pm 16 \%$ and $69 \pm 17 \%$ of nonoxidative glucose metabolism in the IDDM and control subjects, respectively (Table I).

Muscle glycogen turnover. Glycogen turnover as determined from the loss of labeled glycogen during the unlabeled-glucose chase period was significantly less $(P<0.05)$ in the IDDM subjects, $16 \pm 4 \%$, than in the control group, $33 \pm 5 \%$. Since glycogen breakdown relative to synthesis was also much less than that of the control subjects, the impaired rate of net glycogen synthesis in the IDDM was the result of a marked reduction in flux through glycogen synthase. Hence, absolute fluxes through both glycogen synthase and phosphorylase are reduced in the IDDM subjects.

Muscle G-6-P. Intramuscular metabolite concentrations observed by ${ }^{31} \mathrm{P}-\mathrm{NMR}$ are reported in Table I and representative spectra are presented in Fig. 2. Basal G-6-P concentrations were similar in the IDDM and control subjects, $0.11 \pm 0.03$ $\mathrm{mmol} / \mathrm{kg}$ of muscle and $0.13 \pm 0.01 \mathrm{mmol} / \mathrm{kg}$ of muscle $(P=$ NS), respectively. Under hyperglycemic-hyperinsulinemic conditions, the mean G-6-P concentration rose in the control subjects to a steady state value of $0.25 \pm 0.01 \mathrm{mmol} / \mathrm{kg}$ of muscle during the clamp which was $\sim 40 \%$ higher than the concentration of $0.18 \pm 0.02 \mathrm{mmol} / \mathrm{kg}$ of muscle observed in the IDDM subjects $(P<0.05)$. During the euglycemic-hyperinsulinemic clamp where the metabolic rate of the controls matched that of the IDDM subjects during hyperglycemic-hyperinsulinemia, the G-6-P concentration fell to $0.18 \pm 0.01 \mathrm{mmol} / \mathrm{kg}$ of muscle in the controls.

\section{Discussion}

To better understand the mechanism of insulin resistance which occurs in patients with IDDM, we measured net rates of muscle glycogen synthesis and turnover (using ${ }^{13} \mathrm{C}-\mathrm{NMR}$ ), and changes in intramuscular G-6-P concentrations (using ${ }^{31} \mathrm{P}-\mathrm{NMR}$ ), in subjects with poorly controlled IDDM and in age-weightmatched healthy control subjects under similar conditions of hyperglycemic-hyperinsulinemia. Plasma concentrations of free
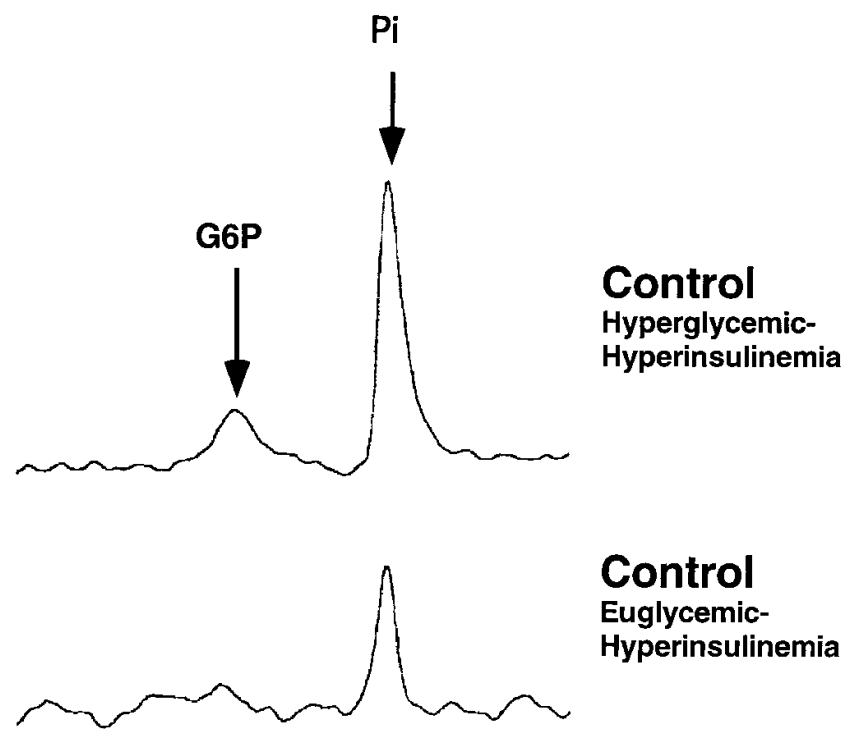

Control EuglycemicHyperinsulinemia

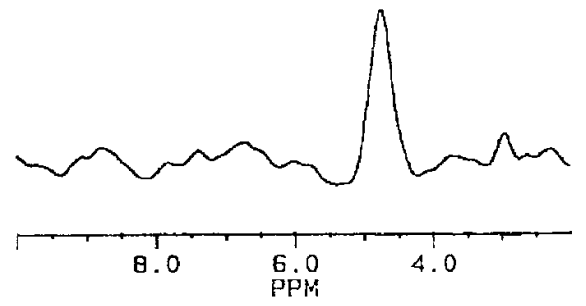

IDDM HyperglycemicHyperinsulinemia

Figure 2. Phosphomonoester $/ \mathrm{P}_{\mathrm{i}}$ region of representative ${ }^{31} \mathrm{P}-\mathrm{NMR}$ difference spectra (clamp minus baseline) of the gastrocnemius muscle of a normal (top) and poorly controlled IDDM (bottom) subject during hyperglycemic-hyperinsulinemia. The middle spectrum is a representative ${ }^{31} \mathrm{P}-\mathrm{NMR}$ difference spectrum (clamp minus baseline) of the gastrocnemius muscle of the same normal subject during euglycemic-hyperinsulinemia when whole body glucose metabolism was similar to the IDDM during hyperglycemic-hyperinsulinemia. 
insulin were matched in both groups at a level that gives about one-half maximal stimulation of muscle glucose uptake. By combining these measurements with rates of whole body oxidative glucose metabolism, the proportion of nonoxidatively metabolized glucose incorporated into muscle glycogen (i.e., net glycogen synthesis) was estimated to be between 70 and $80 \%$ in both groups. The remainder of nonoxidative glucose disposal could be accounted for by liver glycogen synthesis (7). Thus, the major factor responsible for diminished rates of insulin-mediated glucose disposal observed in patients with poorly controlled IDDM is a defect in muscle glycogen synthesis.

Net rates of glycogen synthesis could be reduced in the IDDM subjects by any of several mechanisms. One possibility might be due to higher rates of glycogen cycling as a result of increased activity of glycogen phosphorylase relative to glycogen synthase. To examine this question we performed $\left[{ }^{13} \mathrm{C}\right] \mathrm{glu}-$ cose pulse, $\left[{ }^{12} \mathrm{C}\right]$ glucose chase studies. As previously discussed, this method will yield a minimal estimate of glycogen cycling since it will not detect any labeled glycogen that cycles down to G-6-P and returns to glycogen (8). Nevertheless, using this approach we calculated rates of glycogen turnover in the healthy controls of $\sim 30 \%$ during times of net glycogen synthesis. In the IDDM subjects, muscle glycogen turnover was decreased by one-half to $\sim 16 \%$. It is unclear what factors account for the decreased glycogen turnover rate in the IDDM subjects. One possibility is that the reduced glycogen phosphorylase flux rate could be a secondary effect of glucose toxicity. Another possibility is that the turnover rate is proportional to glycogen concentration, and that the concentration of muscle glycogen in the IDDM subjects is less than that of the controls. To discriminate between these possibilities, it would be necessary to obtain turnover rates in healthy controls at different muscle glycogen concentrations and at concentrations matched to that of the IDDM subjects. However, it is clear from this study that the decrease in net glycogen synthesis in the IDDM subjects was not the result of greater glycogen cycling, but rather due to decreased flux through glycogen synthase.

To determine other biochemical factors underlying the reduced rates of glycogen synthesis in the diabetic subjects, we used ${ }^{31} \mathrm{P}-\mathrm{NMR}$ to measure intracellular concentrations of G-6-P in the subjects before and during the hyperglycemic-hyperinsulinemic clamp and during a euglycemic-hyperglycemic clamp in the control subjects to match their whole body glucose metabolism with that of the IDDM subjects. Analysis of the correlation of the glycogen synthesis rate with the incremental change in G-6-P makes it possible to determine the rate-controlling step for glycogen synthesis $(10,15)$. The increment in G-6-P concentration in the IDDM subjects in response to the glucose infusion was about half of that seen in the controls. With similar rates of nonoxidative glucose disposal, there was no significant difference in the G-6-P concentration between the groups. If glycogen synthase were rate-limiting, a buildup of G-6-P would be expected concomitant with the reduced rates of glycogen synthesis seen in the diabetic subjects. Since the increment in intramuscular G-6-P concentration was lower in the IDDM subjects when their nonoxidative glucose disposal was also lower than the healthy controls, and since matching nonoxidative glucose disposal resulted in equivalent G-6-P concentrations in both groups, it is clear that either glucose transport and/or phosphorylation is rate-controlling for glycogen synthesis and is impaired in the diabetic subjects.
While it is theoretically possible that an increased rate of glycolysis could explain the smaller increment in G-6-P concentration in the IDDM subjects, this is very unlikely since any increase in glycolytic lactate production would be included in the measured rate of nonoxidative glucose metabolism which was $\sim 50 \%$ lower in the IDDM subjects. Furthermore, the lower concentrations of blood lactate observed in diabetic subjects during the hyperglycemic-hyperinsulinemic clamp suggest that rates of glycolysis were (if anything) lower in the diabetic subjects than the control subjects. Finally, a higher rate of oxidative glycolysis would be reflected by an increase in rates of glucose oxidation which were not significantly different between groups. Our observations are consistent with the hypothesis that insulin resistance in patients with poorly controlled IDDM is due to desensitization of the glucose transport system by elevated intramuscular concentrations of hexosamine and is consistent with some recent studies in rodents. Baron et al. have shown that an infusion of glucosamine during euglycemic-hyperglycemic clamps in awake rats resulted in an $\sim 40 \%$ decrease in the translocation of GLUT4 transporters to the muscle membrane and an $\sim 33 \%$ reduction in glucose uptake compared with the control animals (5). In another study Herber and McClain created a transgenic mouse model which overexpresses the GFAT enzyme and demonstrated that these mice were insulin resistant as reflected by relative hyperinsulinemia in the fed state (16).

In summary, under hyperglycemic-hyperinsulinemic conditions whole body glucose metabolism was reduced by $\sim 40 \%$ in the IDDM subjects compared with age-weight-matched control subjects. The reduction in whole body glucose metabolism in the diabetic subjects could be attributed to a reduced rate of muscle glycogen synthesis, which accounted for most of the insulin-stimulated glucose disposal in both groups. Net rates of muscle glycogen synthesis in the IDDM subjects were only about half that of the healthy controls and accounted for most of the decrease in overall nonoxidative glucose disposal in these subjects. The reduced rate of net glycogen synthesis in the IDDM subjects was due to a marked reduction in glycogen synthase flux as opposed to an increase in glycogen cycling. Furthermore, from the ${ }^{31} \mathrm{P}-\mathrm{NMR}$ observation of a blunted increase in intramuscular G-6-P concentration in the diabetic subjects during the clamp, we determined that the major reason for this reduced rate of muscle glycogen synthesis can be attributed to a defect in glucose transport/phosphorylation.

\section{Acknowledgments}

We would like to thank the nurses and staff of the Yale New Haven Hospital General Clinical Research Center for their help in performing these studies and Veronika Walton for technical assistance.

This work was supported by grants from the Public Health Service: RO1 DK-49230, PO1 DK-45735, M01 RR-00123-26, and a grant from the Juvenile Diabetes Foundation, International (G.I. Shulman).

\section{References}

1. Revers, R.R., O.G. Kolterman, J.A. Scarlett, R.S. Gray, and J.M. Olefsky. 1984. Lack of in vivo insulin resistance in controlled insulin-dependent, type I, diabetic patients. J. Clin. Endocrinol. Metab. 58:353-358.

2. Yki-Järvinen, H., E. Helve, and V.A. Koivisto. 1987. Hyperglycemia decreases glucose uptake in type I diabetes. Diabetes. 36:892-896.

3. Rossetti, L., A. Giaccari, and R.A. DeFronzo. 1990. Glucose toxicity. Diabetes Care. 13:610-630.

4. Marshall, S., V. Bacate, and R.R. Traxinger. 1991. Discovery of a meta- 
bolic pathway mediating glucose-induced desensitization of the glucose transport system. J. Biol. Chem. 266:4706-4712.

5. Baron, A.D., J.-S. Zhu, J.-H. Zhu, H. Weldon, L. Maianu, and W.T. Garvey. 1995. Glucosamine induces insulin resistance in vivo by affecting GLUT4 translocation in skeletal muscle. Implications for glucose toxicity. $J$. Clin. Invest. 96:2792-2801.

6. Shulman, G.I., D.L. Rothman, T. Jue, P. Stein, R.A. DeFronzo, and R.G. Shulman. 1990. Quantitation of muscle glycogen synthesis in normal subjects and subjects with non-insulin-dependent diabetes by ${ }^{13} \mathrm{C}$ nuclear magnetic resonance spectroscopy. N. Engl. J. Med. 322:223-228.

7. Cline, G.W., D.L. Rothman, I. Magnusson, L.D. Katz, and G.I. Shulman. 1994. ${ }^{13} \mathrm{C}$-nuclear magnetic resonance spectroscopy studies of hepatic glucose metabolism in normal subjects and subjects with insulin-dependent diabetes mellitus. J. Clin. Invest. 94:2369-2376.

8. Magnusson, I. D.L. Rothman, B. Jucker, G.W. Cline, R.G. Shulman, and G.I. Shulman. 1994. Liver glycogen turnover in fed and fasted humans. Am. J. Physiol. 266(Endocrinol. Metab. 29):E796-E803.

9. Rothman, D.L., R.G. Shulman, and G.I. Shulman. 1992. ${ }^{31} \mathrm{P}$-nuclear magnetic resonance measurements of muscle glucose-6-phosphate. Evidence for reduced insulin-dependent muscle glucose transport or phosphorylation activity in non-insulin-dependent diabetes mellitus. J. Clin. Invest. 89:1069-1075.

10. Rothman, D.L., I. Magnusson, G. Cline, D. Gerard, C.R. Kahn, R.G.
Shulman, and G.I. Shulman. 1995. Decreased muscle glucose transport/phosphorylation is an early defect in the pathogenesis of non-insulin-dependent diabetes mellitus. Proc. Natl. Acad. Sci. USA. 92:983-987.

11. DeFronzo, R.A., J.E. Tobin, and R. Andres. 1979. Glucose clamp techniques: a method for quantifying insulin secretion and resistance. Am. J. Physiol. 237(Endocrinol. Metab.)E214-E223.

12. Taylor, R., T.B. Price, D.L. Rothman, R.G. Shulman, and G.I. Shulman. 1992. Validation of ${ }^{13} \mathrm{C}$ NMR measurement of human muscle glycogen by comparison with biopsy and direct biochemical measurement. Magn. Reson. Med. 27:13-20.

13. Bloch, G., J.R. Chase, M.J. Avison, and R.G. Shulman. 1993. In vivo ${ }^{31} \mathrm{P}$ NMR measurement of glucose-6-phosphate in the rat muscle after exercise. Magn. Reson. Med. 30:347-350.

14. Cohn, S.H., D. Vartsky, S. Yasumura, A. Sawitsky, I. Zanzi, A. Vaswani, and K.J. Ellis. 1980. Compartmental body composition based on total-body nitrogen, potassium, and calcium. Am. J. Physiol. 239:E524-E530.

15. Shulman, R.G., G. Bloch, and D.L. Rothman. 1995. In vivo regulation of muscle glycogen synthase and the control of glycogen synthesis. Proc. Natl. Acad. Sci. USA. 92:8535-8542.

16. Herber, L.F., and D.A. McClain. 1995. Overexpression of glutamine: fructose-6-phosphate in skeletal muscle of transgenic mice results in insulin resistance. Diabetes. 44:15A. 